\title{
Artgerecht! - Wie gesund ist die vegetarische und vegane Ernährung von Hund und Katze?
}

\author{
Cornelia Rückert
}

Manch ein Besitzer entscheidet sich heute für eine „bewusste“ vegetarische oder vegane Ernährung von Hund und Katze. Wir beantworten die 7 häufigsten Fragen rund um diese Ernährungsform.

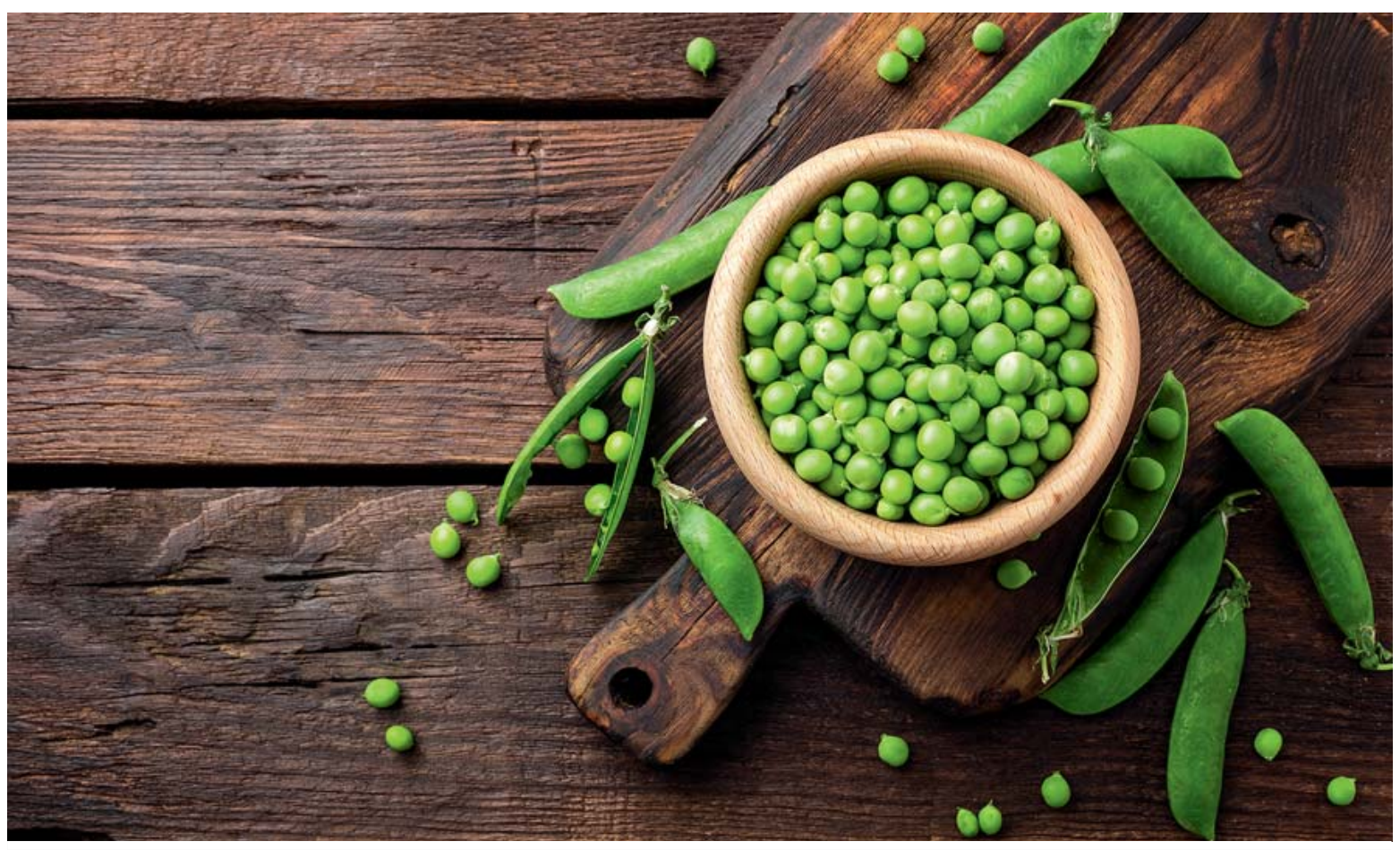

Quelle: @ Sea Wave - stock.adobe.com

In den letzten Jahren orientierten sich viele Hunde- und Katzenhalter bezüglich der Ernährung ihres Vierbeiners sehr stark an den wilden Vorfahren ihrer Tiere und präferierten eine fleischbetonte Fütterung. Seit Kurzem kommt nun der Trend aus der Humanernährung, verstärkt auf tierische Produkte zu verzichten, auch in der Fütterung unserer Hunde und Katzen an. Im folgenden Artikel finden Sie die wichtigsten Antworten auf häufig gestellte Fragen zu diesem Thema.
Ist es möglich, meinen Hund vegetarisch zu ernähren?

Bei der vegetarischen Fütterungsform wird auf Fleisch und Fisch verzichtet, jedoch sind tierische Proteinquellen wie Milchprodukte (Frischkäse, Quark, Joghurt etc.) und Ei erwünscht. Sie decken einen hohen Anteil des Proteinbedarfs ab. Ergänzt werden sie durch Kohlenhydrate zur Energiebedarfsdeckung sowie pflanzliche Öle, Obst und/ oder Gemüse und ein geeignetes vitaminisiertes Mineral- 
futter. Diese Form der Ernährung ist für den Hund grundsätzlich möglich und nichts spricht dagegen, ab und an eine vegetarische Mahlzeit anzubieten. Sind die Beweggründe des Tierhalters jedoch vom eigenen ethischen Empfinden auf den Hund übertragen, sollte das Tierschutzgesetz nichts ganz außer Acht gelassen werden.

Hier steht im 2. Abschnitt in §2:

„Wer ein Tier hält, betreut oder zu betreuen hat,

1. muss das Tier seiner Art und seinen Bedürfnissen entsprechend angemessen ernähren, pflegen und verhaltensgerecht unterbringen, $[\ldots]$ “..

Dies beinhaltet, dass unsere Hunde als Karni-Omnivoren, also fleischbetonte Allesfresser, nicht ohne triftigen Grund dauerhaft vegetarisch gefüttert werden sollten.

\section{Fälle, in denen eine vegetarische Fütterung angezeigt ist}

Hier sind vor allem Futtermittelallergien zu nennen, bei denen der betreffende Hund kaum noch eine Fleischsorte verträgt. In diesem Fall kann ein Ausweichen auf Milchprodukte oder pflanzliche Proteinträger eine gute Lösung darstellen. Beachtet werden sollte hier jedoch, dass Hunde, die auf zahlreiche Proteinquellen allergisch reagieren, häufig nach einem gewissen Zeitraum auch die neu eingeführten, bisher gut akzeptierten Proteinkomponenten nicht mehr vertragen.

Ein weiteres Beispiel für die häufige Anwendung von Rationen, die stark fleischreduziert sind oder ganz auf Milchprodukte als Proteinquellen setzen, sind erworbene Lebererkrankungen. Auch der kongenitale portosystemische Shunt, bei dem sich die Patientenbesitzer gegen eine chirurgische Lösung entscheiden, ist ein häufiger Grund. Bei Humanpatienten mit einem im Zuge des portosystemischen Shunts auftretendem hepatoenzephalen Syndrom wurden Proteine aus Sojaisolaten und Milchprodukten bedeutend besser vertragen. Die gleichen Ergebnisse werden auch für den Hund beschrieben.

\section{Welche Futtermittel sind für eine vegetarische Fütterung geeignet?}

Bei der Verfütterung größerer Mengen von Milchprodukten sollte die Laktoseverträglichkeit des Hundes beachtet werden, die jedoch individuell sehr differieren kann. Verschiedene Hartkäse können aufgrund ihres vergleichsweise niedrigen Laktosegehalts auch in größeren Mengen eingesetzt werden. Zu beachten ist jedoch der recht hohe Fett- und somit Energiegehalt der meisten Sorten. Setzt man beispielsweise Käsesorten wie Emmentaler,
\Tab. 1 Kalziumgehalte in ausgewählten Lebensmitteln.

\begin{tabular}{|c|c|}
\hline Lebensmittel & $\begin{array}{l}\text { Kalziumgehalt } \\
\text { in } \mathrm{mg} / 100 \mathrm{~g} \text { us }\end{array}$ \\
\hline Joghurt (3,5\% Fett) & 120 \\
\hline Vollmilch (Rind) & 115 \\
\hline Frischkäse (20\% Fett in der TS²) & 85 \\
\hline Magerquark & 70 \\
\hline Vollei (ohne Schale) & 50 \\
\hline
\end{tabular}

Edamer oder Gouda zur Deckung des Proteinbedarfs ein, ist hiermit der Energiebedarf eines adulten durchschnittlich aktiven Hundes ebenfalls gedeckt. Eine Ergänzung mit einer Kohlenhydrat- oder Fettquelle ist dabei nicht nötig. Ebenfalls laktosearme, aber fett- und somit energieärmere Produkte sind Magerquark und Hüttenkäse (körniger Frischkäse). Bei den meisten Hunden zeichnen sie sich durch eine hohe Verträglichkeit aus, sodass sie häufig bei diätetischen Patienten eingesetzt werden.

Im Gegensatz zu den Milchprodukten sind Eier kohlenhydratfrei und enthalten v. a. Protein und Fett. Sie werden von Hunden meist gern akzeptiert und sind dabei gut verdaulich. Auch der hohe Gehalt an essenziellen Aminosäuren und fettlöslichen Vitaminen im Eidotter machen Eier zu einem geeigneten Futtermittel in der Hundeernährung. Aufgrund einer möglichen Salmonellenbelastung und des in rohen Eiern hohen Anteils an Trypsinhemmstoffen und Avidin (bindet Biotin) sollten Eier jedoch immer ausreichend erhitzt angeboten werden.

\section{Vegetarische Ration: Lässt sich der Kalziumbedarf über Milchprodukte decken?}

In der Humanernährung werden Milchprodukte zur Deckung des Kalziumbedarfs empfohlen. Die Deutsche Gesellschaft für Ernährung empfiehlt hier 1000 mg Kalzium/ Tag für einen durchschnittlichen Erwachsenen $(\sim 80 \mathrm{~kg})$. In Anbetracht der Kalziumgehalte der in der vegetarischen Ernährung häufig eingesetzten Lebensmittel lässt sich dieser Bedarf mit einem ausgewogenen Ernährungsstil leicht decken ( $\triangleright$ Tab. 1 ).

Im Unterschied zum Menschen haben Hunde aber einen ungleich höheren Kalziumbedarf. Dieser wird mit $130 \mathrm{mg} / \mathrm{kg} \mathrm{KM}^{0,75} / \mathrm{Tag}$ angegeben. Ein $20 \mathrm{~kg}$ schwerer Hund benötigt somit $1230 \mathrm{mg}$ Kalzium/Tag. Dieser Bedarf wäre theoretisch mit einer Tagesration, deren Proteinquellen aus 2 Eiern ( $120 \mathrm{~g} \mathrm{Ei}), 600 \mathrm{~g}$ Magerquark und $600 \mathrm{~g}$ Joghurt (3,5\% Fett) zusammengesetzt sind, gedeckt. Diese Form der Fütterung würde jedoch mit 
einer immensen Überschreitung des Protein- und Energiebedarfs einhergehen und ist daher nicht zu empfehlen. Senkt man die Menge der Proteinträger somit auf den Bedarf des Hundes herab, sollte ein geeignetes Mineralfuttermittel ergänzt werden.

Bei der Katze liegt der Kalziumbedarf mit $71 \mathrm{mg} / \mathrm{kg}$ $\mathrm{KM}^{0,67} /$ Tag unter dem des Hundes. Jedoch ist auch hier aufgrund der eingeschränkten Laktosetoleranz eine Kalziumbedarfsdeckung über Milchprodukte nicht möglich.

\section{Wie unterscheidet sich dazu die vegane Ernährungsform?}

Hier wird komplett auf Proteinträger tierischen Ursprungs verzichtet. Stattdessen werden Hülsenfrüchte (Bohnen, Erbsen, Linsen, Lupinen), Sojaprodukte (Tofu, SojaSchnetzel) oder auch Seitan (Weizeneiweiß) und Nüsse oder Saaten eingesetzt. Ebenso werden hier zur Energiebedarfsdeckung verschiedenste Kohlenhydratquellen eingesetzt und die Ration mit Obst und/oder Gemüse und pflanzlichen Ölen ergänzt.

\section{Ist die Wertigkeit pflanzlicher Protein- quellen mit tierischen vergleichbar?}

Die biologische Wertigkeit beschreibt das Verhältnis zwischen resorbiertem und retiniertem Stickstoff und charakterisiert somit die intermediäre Verfügbarkeit des aufgenommenen Proteins. Hiermit haben vor allem die Proteine eine hohe biologische Wertigkeit, deren Aminosäuremuster dem zu synthetisierenden Protein möglichst nahekommt. Die biologische Wertigkeit wird als Prozentzahl angegeben und ist tierartspezifisch unterschiedlich.

\section{Merke}

Fehlen hierbei einzelne essenzielle Aminosäuren, wird die Gesamtproteinsynthese eingeschränkt.

Für Hund und Katze essenzielle Aminosäuren sind:

- Histidin

- Lysin

- Leucin

- Isoleucin

- Valin

- Threonin

- Tryptophan

- Arginin

- Methionin (teils durch Cystein ersetzbar)

- Phenylalanin (teils durch Tyrosin ersetzbar)

- bei der Katze zusätzlich: Taurin (Aminosulfonsäure)

In $>$ Abb. 1 sind die biologische Wertigkeit tierischer und pflanzlicher Proteinquellen für den Hund aufgezeigt.
Um den Bedarf des Hundes an den einzelnen essenziellen Aminosäuren mit den Gehalten in fleischbetonten, vegetarischen und veganen Rationen zu vergleichen, wurden zunächst - stellvertretend für die unterschiedlichen Fütterungsstile - 3 Beispielrationen entworfen ( $>$ Tab. 2).

Wie in $\mathbf{A b b} \mathbf{2}$ ersichtlich, ist sowohl in fleischhaltigen wie auch in vegetarischen Rationen (Basis der Kalkulation sind die Beispielrationen aus $~$ Tab. 2) eine komplette Bedarfsdeckung mit allen essenziellen Aminosäuren gegeben. Bei der veganen Beispielration ist der Methioninbedarf jedoch nur zu 62\% gedeckt. Nach dem Prinzip der biologischen Wertigkeit wird hiermit auch die Verfügbarkeit der restlichen Aminosäuren eingeschränkt. Bei einer zumindest teilweisen Deckung des Proteinbedarfs über Sojaprodukte kann die Methioninlücke hier jedoch geschlossen werden.

\section{Welche weiteren Probleme können sich bei veganen Rationen ergeben?}

Als Proteinträger werden Sojaprodukte, aber auch Linsen oder Leguminosenfrüchte (Erbsen, Bohnen, Lupinen etc.) eingesetzt. Sie enthalten in der Nährstofffraktion der stickstofffreien Extraktstoffe (NfE) neben Stärke auch Oligosaccharide (v.a. Stachyose und Raffinose). Diese sind im Dünndarm weitgehend unverdaulich und werden daher im Dickdarm fermentiert, womit das Risiko für weichen Kot und Flatulenzen einhergeht. Daher wird in der Literatur meist eine Mengenbegrenzung von Erbsen und Bohnen auf 5-10\% der Gesamtration empfohlen.

Ein bedeutender Engpass kann bei veganer Fütterung auch in der Versorgung mit B-Vitaminen bestehen, insbesondere von Vitamin B12. Dies ist der Fall, wenn diese nicht durch ein geeignetes Ergänzungsfuttermittel supplementiert werden. Zur Bedarfsdeckung an Vitamin B12 wird häufig Bierhefe eingesetzt. Aus der Humandiätetik gibt es jedoch aktuell Zweifel daran, ob das Vitamin B12 aus Bierhefe für den menschlichen Organismus bioverfügbar ist. Für den Hund liegen zu dieser Fragestellung aktuell keine Daten vor.

Beachtung sollte auch der hohe Gehalt an Phytinsäure in pflanzlichen Stärke- und Proteinträgern finden. Phytinsäure bindet Phosphor - Fleischfresser besitzen nur eine geringe Menge körpereigener Enzyme, um diese Verbindung zu lösen und den Phosphor somit für den Organismus verfügbar zu machen.

\section{Wie verhält es sich mit dem hochspezialisierten Karnivor Katze?}

Während eine ovo-lakto-vegetarische Fütterung des Hundes grundsätzlich möglich ist, sind hier bei der Katze 


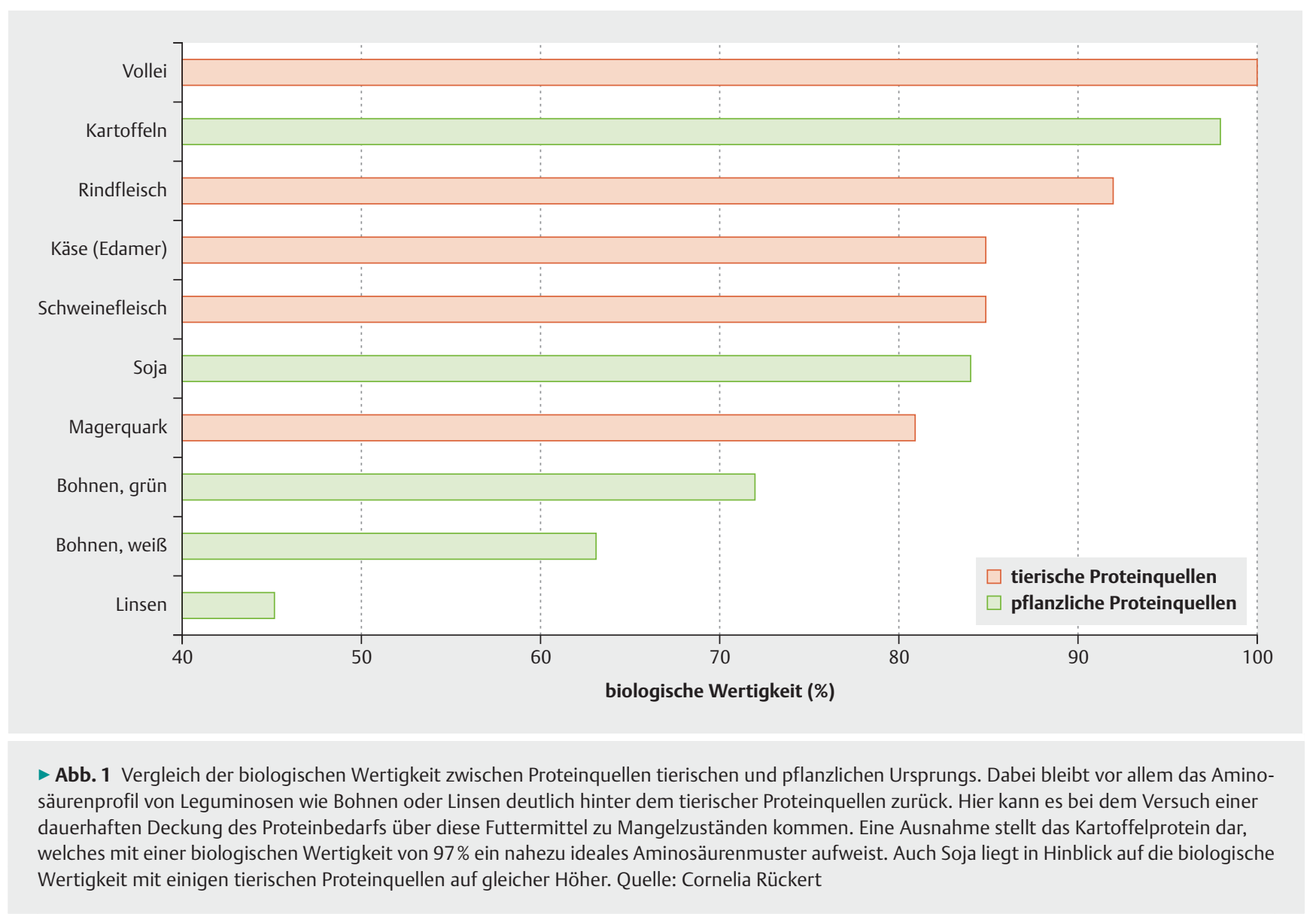

bereits deutliche Grenzen gesetzt. Zum einen unterliegen Katzen einer starken Nahrungsprägung. Futtermittel, die im Jungtieralter von der Katze kennengelernt und akzeptiert wurden, stellen auch später meist kein Problem in der Akzeptanz dar. Es ist daher fraglich, ob eine Katze eine so drastische Umstellung der Einzelfutterkomponenten überhaupt annimmt.

Zudem haben Katzen eine sehr eingeschränkte Verdauungskapazität von Kohlenhydraten, zu denen auch Laktose gehört. Laktosehaltige Milchprodukte sollten daher nur in begrenzten Mengen angeboten werden. Setzt man jedoch eine Laktoseverträglichkeit von maximal $2 \mathrm{~g} / \mathrm{kg}$ an, so befindet man sich mit einer bedarfsdeckenden Ration meist noch im Rahmen dieser Toleranzgrenze.

Für eine $4 \mathrm{~kg}$ schwere Katze könnte folgende Beispielration (Tagesmenge) erstellt werden:

- $60 \mathrm{~g}$ gekochtes Ei

- $80 \mathrm{~g}$ körniger Frischkäse (20\% Fett in der Trockensubstanz)

- $2,5 \mathrm{~g}$ Leinöl

- vitaminisiertes Mineralfutter (speziell für Katzen)

Körniger Frischkäse hat einen durchschnittlichen Laktosegehalt von $\sim 3 \mathrm{~g} / 100 \mathrm{~g}$, in der aufgezeigten Ration sind dementsprechend $\sim 2,4 \mathrm{~g}$ Laktose enthalten. Dieser
Wert liegt innerhalb der Toleranzgrenze von maximal $8 \mathrm{~g}$ Laktose für eine $4 \mathrm{~kg}$ schwere Katze. Verzichtet man auf die Fütterung von Ei und möchte den Proteinbedarf dieser Katze komplett mit körnigem Frischkäse decken, würde sich die Menge hier auf $\sim 150 \mathrm{~g} /$ Tag erhöhen. Selbst mit dieser Laktoseaufnahme von $4,5 \mathrm{~g}$ liegt die Katze noch innerhalb ihrer Verdauungskapazität. Fraglich bleibt jedoch die bereits oben erwähnte Akzeptanz der fleischlosen Ration.

Weiterhin gilt es unter Berücksichtigung des strikt karnivoren Stoffwechsels der Katze zusätzlich besondere Ansprüche zu beachten: So ist die Aufnahme der $\beta$-Aminosulfonsäure Taurin für die Katze essenziell. Diese ist nur in tierischen Geweben vorhanden. Die Empfehlung zur täglichen Taurinversorgung beträgt $0,1 \mathrm{~g} / 1000 \mathrm{kcal} \mathrm{ME}$ [1]. In einer fleischbasierten Ration wird dieser Bedarf auch ohne ein zusätzliches Taurin-Supplement gedeckt, in vegetarischen Rationen ist dies jedoch nicht möglich. Zwar enthalten sowohl Eier wie auch Milchprodukte geringe Mengen an Taurin, diese Gehalte bleiben jedoch weit hinter denen von Fleisch und tierischen Nebenprodukten zurück.

Auch die Fettsäure Arachidonsäure ist für Katzen essenziell. Der Bedarf kann somit nicht über eine vegetarische Ration gedeckt werden, da diese Fettsäure nur in tieri- 


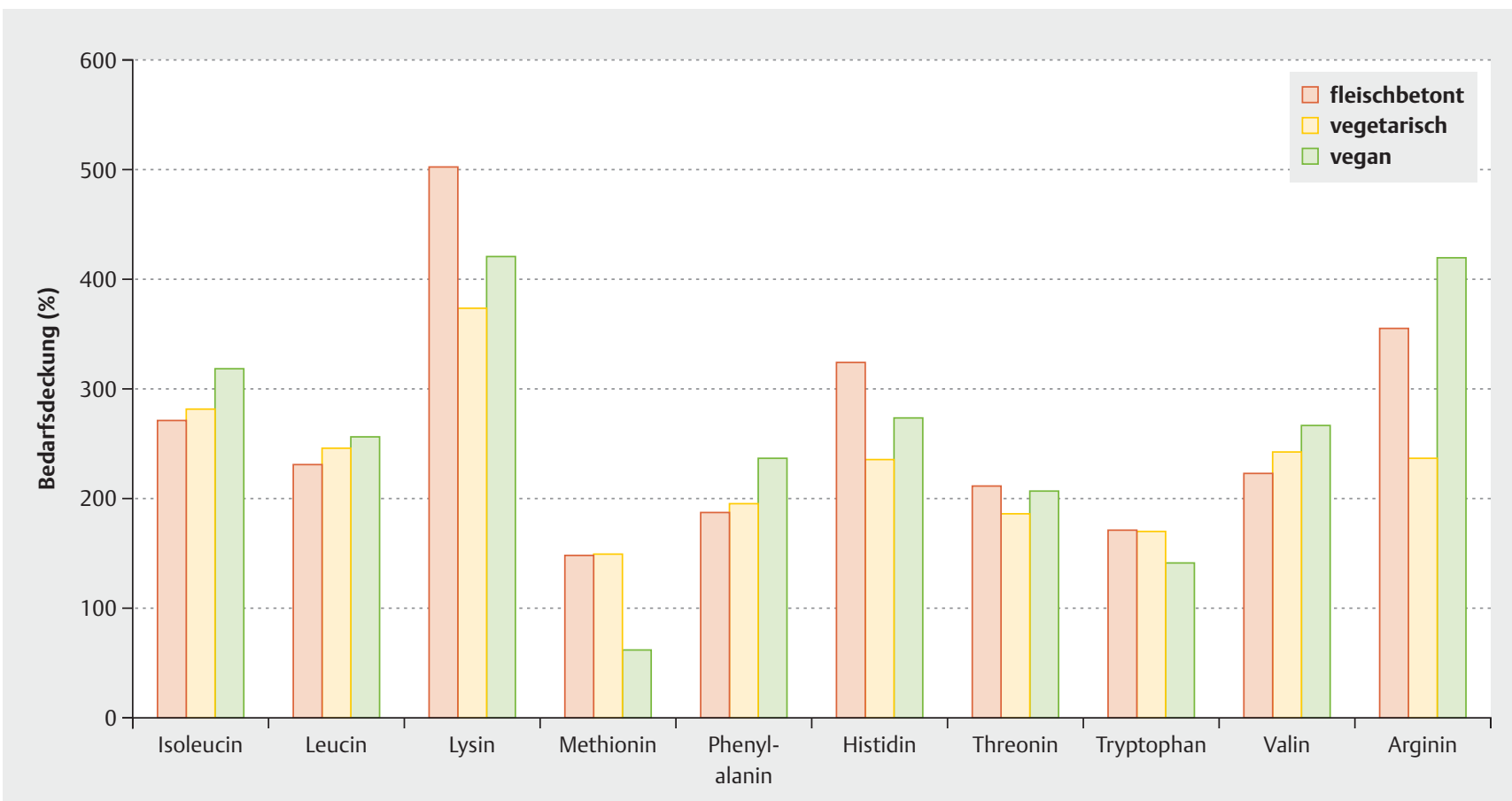

Abb. 2 Vergleich zwischen einer fleischbetonten, einer vegetarischen und einer veganen Beispielration in Bezug auf die Bedarfsdeckung mit essenziellen Aminosäuren. Quelle: Cornelia Rückert

- Tab. 2 Beispielrationen für einen adulten 15 kg schweren Hund (Basis: Protein- und Energiebedarfsdeckung; alle Mengenangaben als Rohgewichte).

\begin{tabular}{|c|c|c|}
\hline fleischbetonte Ration & vegetarische Ration & vegane Ration \\
\hline $\begin{array}{l}\text {. } 180 \mathrm{~g} \text { Rindfleisch, Keule } \\
\text { " } 10 \mathrm{~g} \text { Leinöl } \\
\text {. } 80 \mathrm{~g} \text { Reis, poliert } \\
\text { " } 100 \mathrm{~g} \text { Möhren } \\
\text { " + vitaminisiertes Mineralfutter* }\end{array}$ & $\begin{array}{l}\text {. } 220 \mathrm{~g} \text { körniger Frischkäse } \\
\text {. } 60 \mathrm{~g} \text { Vollei } \\
\text { " } 10 \mathrm{~g} \text { Leinöl } \\
\text {. } 90 \mathrm{~g} \text { Reis } \\
\text {. } 100 \mathrm{~g} \text { Möhren } \\
\text {. + vitaminisiertes Mineralfutter* }\end{array}$ & $\begin{array}{l}\text { - } 100 \mathrm{~g} \text { Gartenbohnen, weiß } \\
\text { - } 100 \mathrm{~g} \text { Linsen } \\
\text { - } 10 \mathrm{~g} \text { Leinöl } \\
\text { " } 50 \mathrm{~g} \text { Reis } \\
\text {. } 80 \mathrm{~g} \text { Möhren } \\
\text { " + vitaminisiertes Mineralfutter* }\end{array}$ \\
\hline
\end{tabular}

schen Fetten enthalten ist. Der Bedarf an den ebenfalls essenziellen Linol- und $\alpha$-Linolensäuren kann jedoch über die Gabe eines pflanzlichen Öles (z. B. Leinöl) gedeckt werden.

Aufgrund der fehlenden Carotinaseaktivität ist die Katze im Gegensatz zum Hund nicht in der Lage, $\beta$-Carotin in Vitamin A umzuwandeln. Während $\beta$-Carotin in pflanzlichen Produkten enthalten ist (diverse Gemüsesorten wie Möhren, Paprika, grünes Blattgemüse), kommt Vitamin A nur in tierischen Produkten - in den höchsten Mengen in der Leber - vor.

Eine vegane Ration, die auf pflanzlichen Proteinquellen wie Sojaprodukten oder heimischen Leguminosefrüchten basiert, wird zudem von einem Großteil der Katzen nicht akzeptiert. Katzen unterliegen einer starken Nahrungs- prägung, die meist schon mit Ende des Welpenalters abgeschlossen ist. Eine Katze hungern zu lassen, um sie letztendlich zur Aufnahme der angebotenen Ration zu zwingen, schließt sich aus. Hier bestünde die Gefahr einer überstürzten Fettmobilisation und einer damit einhergehenden fettigen Leberdegeneration, welche mit einer akuten Lebensgefahr für das betreffende Tier einhergeht.

\section{Fazit}

Grundsätzlich sollte eine vegetarische oder vegane Fütterung von Hunden und Katzen kritisch hinterfragt werden - dies gilt vor allem auch für die Beweggründe des Besitzers, diese Fütterungsform einer fleischhaltigen vorzuziehen. Eine ovo-lakto-vegetarische Fütterung von Hunden ist grundsätzlich möglich, bei der Katze können Akzeptanzprobleme auftreten. Eine rein vegane Fütterung des 


\section{TAKE HOME}

- Hunde sind fleischbetonte Allesfresser, Katzen sind hochspezialisierte Karnivoren.

- Eine dauerhafte vegetarische Ernährung bietet sich bei Hunden nur in Einzelfällen an, z. B. bei Futtermittelallergien, erworbenen Lebererkrankungen und kongenitalen portosystemischen Shunts.

- Eine vegane Ernährung kann einen Mangel an Methionin und Vitamin B sowie eine unzureichende Phosphorverfügbarkeit bewirken. Die vegane Ernährung von Katzen ist abzulehnen.

- Bei einer unpassenden Rationszusammenstellung (z. B. hoher Leguminosenanteil) können die Tiere weichen Kot und Flatulenzen entwickeln.

- Bei der Katze können bei einer ovo-lakto-vegetarischen Ernährung deutliche Akzeptanzprobleme aufgrund der starken Nahrungsprägung entstehen. Zudem besteht das Risiko eines Mangels an Taurin, Arachidonsäure und Vitamin A, sodass eine Nahrungsergänzung erforderlich ist.
Hundes sollte genauestens kalkuliert werden, um Nährstoffengpässe zu vermeiden. Bei der Katze ist diese Ernährungsform sowohl aus ernährungsphysiologischer wie auch aus Tierschutzsicht abzulehnen.

Korrespondenzadresse

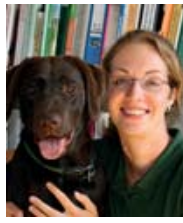

Dr. Cornelia Rückert

Fachtierärztin für Tierernährung und Diätetik Landwirtschaftliche Kommunikations- und Servicegesellschaft $\mathrm{mbH}$

August-Bebel-Str. 6

09577 Niederwiesa

cornelia.rueckert@lks-mbh.com

\section{Literatur}

[1] Subcommittee on Dog and Cat Nutrition. Nutrient Requirements of Dogs and Cats. Washington: National Academies Press; 2006

Bibliografie

DOI https://doi.org/10.1055/a-0902-2755

Kleintier konkret 2019; 22: 18-23

(c) Georg Thieme Verlag KG Stuttgart · New York

ISSN 1434-9132 